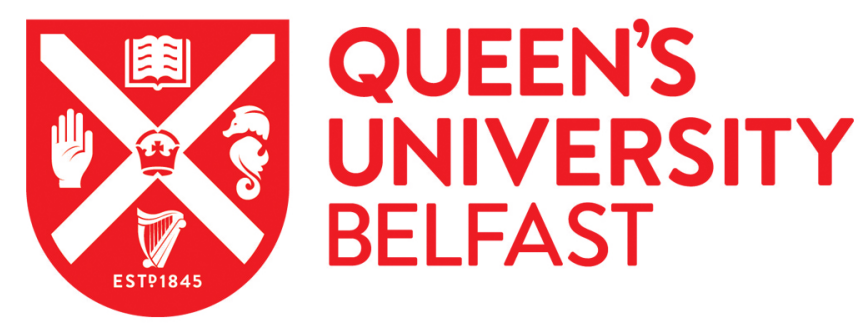

\title{
Attitudes towards worm egg counts and targeted selective treatment against equine cyathostomins
}

Rose Vineer, H., Vande Velde, F., Bull, K., Claerebout, E., \& Morgan, E. R. (2017). Attitudes towards worm egg counts and targeted selective treatment against equine cyathostomins. Preventive veterinary medicine, 144, 6674. https://doi.org/10.1016/j.prevetmed.2017.05.002

Published in:

Preventive veterinary medicine

Document Version:

Peer reviewed version

Queen's University Belfast - Research Portal:

Link to publication record in Queen's University Belfast Research Portal

Publisher rights

(c) 2017 Elsevier B.V. All rights reserved.

This manuscript version is made available under the CC-BY-NC-ND 4.0 license http://creativecommons.org/licenses/by-nc-nd/4.0/,which permits distribution and reproduction for noncommercial purposes, provided the author and source are cited

\section{General rights}

Copyright for the publications made accessible via the Queen's University Belfast Research Portal is retained by the author(s) and / or other copyright owners and it is a condition of accessing these publications that users recognise and abide by the legal requirements associated with these rights.

Take down policy

The Research Portal is Queen's institutional repository that provides access to Queen's research output. Every effort has been made to ensure that content in the Research Portal does not infringe any person's rights, or applicable UK laws. If you discover content in the Research Portal that you believe breaches copyright or violates any law, please contact openaccess@qub.ac.uk. 
1 Attitudes towards worm egg counts and targeted selective

2 treatment against equine cyathostomins

3 Rose Vineer, $H_{.}^{a, b}$, Vande Velde, F. ${ }^{c, d}$, Bull, K. ${ }^{a}$, Claerebout, E.

4 Morgan, E. R..$^{\mathrm{a}, \mathrm{b}}$

5 a School of Veterinary Sciences, University of Bristol, Langford House, Bristol, UK,

$6 \quad$ BS40 5DU

$7 \quad$ b Cabot Institute, University of Bristol, Cantocks Close, Bristol, UK, BS8 1TS

$8{ }^{\mathrm{c}}$ Department of Virology, Parasitology and Immunology, Faculty of Veterinary

9 Medicine, Ghent University, Salisburylaan 133, 9820 Merelbeke, Belgium

10 d Department of Communication Studies, Faculty of Political and Social Sciences,

11 Ghent University, Korte Meer 7-11, 9000 Gent, Belgium 
Abstract

17 Gastrointestinal nematodes present a major threat to the health and welfare of

18 equids worldwide. Anthelmintic resistance (AR) is increasingly reported and

19 challenges effective control in horses and ponies in many regions. The use of faecal

20 worm egg counts (FECs) to support targeted treatment (FEC-TT) and targeted

21 selective treatment (FEC-TST) has been promoted as an effective deworming

22 strategy that may prolong the useful life of anthelmintics and reduce the costs associated with parasite control. However, treatment applied at set intervals or on pre-determined dates remains common. A structural equation model was developed to identify factors influencing the uptake of FEC-directed treatment strategies, based on well-established socio-psychological theories of intentional health-related behaviours: the Theory of Planned Behaviour and the Health Belief Model. More than 850 valid responses were received from horse owners in the UK via an online survey. The intention to use FECs prior to deworming was not influenced by the perceived risk of anthelmintic resistance or that of gastrointestinal nematode infection but was positively influenced by a negative attitude towards anthelmintics, a positive attitude towards FECs, an increase in social pressure (e.g. significant others think the respondent should use FECs) and an increase in perceived control over their deworming programme. The results were consistent with a similar study conducted on FEC-TT in cattle in Belgium. An increase in respondents' selfperceived level of knowledge significantly increased the intention to use FECs via mediating factors. These results suggest that knowledge transfer activities aimed at

38 increasing awareness and understanding of sustainable nematode control practices may be more effective at encouraging behavior change than emphasising the 
41 this study population.

43 Keywords: equine cyathostomins; faecal egg count; structural equation model;

44 theory of planned behavior; health belief model

\footnotetext{
${ }^{1}$ Abbreviations:

UK - United Kingdom

GB - Great Britain

AR - Anthelmintic Resistance

GIN - gastrointestinal nematode

FEC - faecal worm egg count

HBM - Health Belief Model

TPB - Theory of Planned Behaviour

EFA - Exploratory factor analysis

CFA - Confirmatory factor analysis

SEM - structural equation model

CFI - Comparative Fit Index

TLI - Tucker-Lewis Index

RMSEA - Root Mean Square Error of Approximation

SRMR - Standardised Root Mean Residual
} 
47 Gastrointestinal nematodes present a major threat to the health and welfare of 48 equids worldwide, and anthelmintic resistance (AR) increasingly compromises

49 control efforts in horses and ponies (Corning, 2009; Matthews and Lester, 2015).

50 The problem of AR in grazing ruminants (Rose et al., 2015) has been addressed by

51 recommending more discriminant application of anthelmintic treatments at group and

52 individual levels (Charlier et al., 2014). In horses, the use of faecal worm egg counts

53 (FECs) to support targeted treatment (FEC-TT) and targeted selective treatment

54 (FEC-TST) has been promoted as an effective deworming strategy that may prolong the useful life of anthelmintics and reduce the costs associated with parasite control (Matthews and Lester, 2015). In Denmark, anthelmintics can only be prescribed for individual horses when supported by parasitological diagnosis such as high faecal egg counts (Nielsen et al., 2006). Elsewhere, however, the use of FECs to inform TT and TST is voluntary, although it does form a key part of new guidelines for the responsible prescription and use of anthelmintics in horses in the UK (AHDA, 2015).

61 Seventy-six percent of respondents to a recent survey of horse owners in the UK (primarily owners in the south of England) reported that they already use FECs to target treatment (Easton et al., 2016). Despite this relatively high self-reported uptake of FEC-TT, the survey indicates that treatment applied at set intervals or on pre-determined dates remains relatively common in the equine industry in the UK. Furthermore, a survey of French veterinarians found that almost half of practitioners never performed FECs prior to treatment (Salle and Cabaret, 2015). Identifying the

68 knowledge, attitudes and practices of horse owners and managers to nematode infections, AR, diagnosis and control will help identify barriers to the uptake of sustainable GIN control strategies such as FEC-TT and FEC-TST. 
Risk management behaviour in animal owners, such as the use of FECs, may be driven by a range of factors including their perception of disease risk, whether they consider the behaviour to be effective, their underlying knowledge of the risks and management options, access to trusted information on the subject, sociodemographic factors and physical attributes of the farm/stables which may limit the possible behaviours (Garforth et al., 2013; Toma et al., 2013; Alarcon et al., 2014). Adopting new technology or strategies to manage animal health, and other constructive behaviour change, requires animal owners to assess the current level of risk and risks associated with adopting/not adopting the new technology or strategy, sometimes based on uncertainty and incomplete knowledge. By identifying the factors driving and preventing the adoption of risk management behaviours, it may be possible to target and address these factors to increase uptake and behaviour change.

Various model frameworks have been proposed to describe the factors driving risk management behaviours. The Theory of Planned Behaviour (TPB; Ajzen, 1991) describes the intention to perform a behaviour as a function of the individual's attitude towards the behaviour, their perceived control over whether or not they perform the behaviour, and peer/societal influences (Ajzen, 1991). The Health Belief Model (HBM; Rosenstock et al., 1988) shares common elements with the TPB but extends the inclusion of potential barriers to performing a behaviour, e.g. positive attitudes towards the risky behaviour, and includes the evaluation of perceived risk (Valeeva et al., 2011; Vande Velde et al., 2015). Although these models are rooted in studies of human health, they have been applied to evaluate animal health management and identify factors influencing farmer behaviour. For example, a survey of pig fattening farms in the Netherlands 
based on the HBM concluded that emphasising the efficacy of risk management strategies such as biosecurity measures and animal health plans may prove more effective than focussing on farmers' perceived risk of disease (Valeeva et al., 2011). The TPB has been used to identify drivers of disease control by English pig producers (Alarcon et al., 2014), and drivers of and barriers to the reduced use of antibiotics by British dairy cattle farmers (Jones et al., 2015). The TPB and HBM have also been combined to evaluate English sheep and pig farmers' attitudes to disease risk management (Garforth et al., 2013) and to identify factors driving the adoption of sustainable GIN control practices on Belgian cattle farms (Vande Velde et al., 2015). The latter were the first to adapt these models to evaluate the use of diagnostics (including FECs) and parasite control behaviour, providing a framework that can be replicated for different livestock sectors and parasites. Here, the framework developed by Vande Velde et al., (2015) was adapted to reflect differences in potential factors influencing behaviour in horse owners compared with cattle farmers and used to identify barriers to the uptake of FEC-TT and FEC-TST by commercial and private equine owners and managers in the UK. 


\section{Methods}

\section{Theoretical framework}

The theoretical framework was based on the work of Vande Velde et al., (2015) on the use of diagnostics before treatment for GINs in dairy cattle in Belgium, and is a combination of elements in the Theory of Planned Behaviour (TPB) and the Health Belief Model (HBM; Figure 1). The model framework is made up of a structural model (Figure 1; Appendix A), comprising factors, or latent variables, which may influence the intention to use FECs before treatment (referred to as constructs). The constructs in the structural model are evaluated using a set of questions, or observed variables (referred to as items) which form the measurement model. These variables are referred to hereafter as items $\mathrm{x}_{1}$ to $\mathrm{x}_{40}$ and are described in full in Appendix A.

The structural model comprised constructs evaluating the intention to use diagnostics (i.e. FECs) prior to treatment, attitudes towards diagnostics (i.e. FECs), attitudes towards anthelmintics, perceived control over the use of diagnostics (i.e. FECs) prior to treatment and subjective norms, as described by Vande Velde et al. (2015). In addition, a perceived risk construct evaluated perceived susceptibility to infection and AR (i.e. the perceived likelihood of infection or developing AR), and perceived severity of infection and AR. The framework was also extended to include the horse owners' perceived knowledge as a construct which may directly or indirectly affect behaviour intention (Figure 1). 


\section{Survey design}

Survey questions for the structural model were based on validated constructs and items used by Vande Velde et al., (2015) which followed the general guidelines for conducting surveys using socio-cognitive models such as the TPB (Francis et al., 2004). Respondents were also asked about their characteristics e.g. type of horse owner and number of horses under their control, in order to describe the sample population and perform external validation. A detailed list of constructs and items forming the measurement model is provided in Appendix $A$.

\section{Data collection}

Although a passport is a legal requirement for all equids in the UK, no central database of horses nor horse owners exists since the removal of the UK National Equine Database in 2012. Therefore, a randomised survey design was not possible and horse and pony owners were surveyed using a self-selecting online survey (SurveyMonkey Inc., Palo Alto, California, USA, www.surveymonkey.com) between 18/12/2015 and 31/01/2016.

The survey was pilot tested on two private horse owners, one lay person and one owner of a commercial equine establishment before online publication. The survey was promoted via social media (Twitter, equine-related Facebook groups and forums). To encourage complete responses, respondents were offered the chance to enter a prize draw for a $£ 100$ Amazon.co.uk voucher by entering their email address on the final page of the survey. The winner was selected by assigning valid responses random numbers and selecting the lowest number. 


\section{External validation}

To verify the external validity of the survey, commercial respondents' characteristics (type of horse owner/establishment) were compared against characteristics of the UK equine industry (Lantra, 2011). It was not possible to validate the ratio of private and commercial respondents as no data exist for comparison.

The distribution of respondents was not tested for complete spatial randomness as no spatial datasets were available to correct for the distribution of the horse population. The distribution of commercial respondents at a county level within the UK was compared against the distribution of commercial equine establishments in the UK (Lantra, 2011) by Chi Square analysis in R 3.2.2 (R Core Team, 2015). The county of origin of each respondent was extracted from the latitude and longitude coordinates and the Ordnance Survey's Boundary Line ${ }^{\mathrm{TM}}$ dataset using the 'Point sampling tool' in QGIS 2.8.1-Wien (QGIS Development Team, 2015).

\section{Internal validation}

The validity of the measurement model was assessed in several ways. First, Cronbach's alpha was used to test for internal consistency of constructs using the alpha function of the psych R package (Revelle, 2015) where $\alpha>0.7$ is good and $\alpha>0.6$ is acceptable (Vande Velde et al., 2015).

Maximum likelihood exploratory factor analysis (EFA) was then conducted on exogenous items in R using the factanal function of the lavaan package (Rosseel, 2012) with an oblique promax rotation (thus allowing for correlation between factors; Costello \& Osbourne, 2005) to evaluate whether the items making up the constructs were consistent with the theoretical constructs on which they were based. Methods 
followed best practice guidelines for EFA (Costello \& Osbourne, 2005). Due to the multi-level structure of the proposed model EFA was conducted twice: once using items $\mathrm{X}_{1}-\mathrm{X}_{6}$ underlying the 'perceived knowledge' construct, and again using items $\mathrm{X}_{7}-$ $\mathrm{X}_{37}$ which form the intermediate constructs that are exogenous to 'behaviour intention' (Table 1; Figure 1).

\section{Structural equation modelling (SEM)}

Structural equation modelling was performed in $\mathrm{R}$ using the sem function in the lavaan package. Regression equations were based on the proposed structural model (Figure 1) and covariance between the constructs relating to perceived risk. Subjective norms and perceived behavioural control were also allowed to covary due to the potential crossloading identified in the EFA. Item variances were fixed to 0 if negative variances were computed and the variance was not significantly different from 0 . Although the Likert and bipolar 7-point scales generate ordinal items, the lavaan package treats these data as numeric and therefore no further adjustments were necessary.

Model fit can be evaluated using a $x^{2}$ test on the observed and expected covariance matrices. However, model performance was evaluated primarily based on an assessment of misclassification of the structural model as described by Saris et al., (2009) using the modificationIndices function in the lavaan package. The function provides an estimated modification index (MI) between endogenous and exogenous variables, and between factors. If the $\mathrm{Ml}$ is greater than 3.84 (equivalent to the $\mathrm{X}^{2}$ critical value corresponding to one degree of freedom at $p<0.05$ ) then the model may be improved by respecifying the parameter in the model (Whittaker, 2012). To 
determine the need for respecification and model modification the context of the change is considered (i.e. is the modification theoretically plausible?) in combination with the expected parameter change (EPC). The EPC indicates the size of the misspecification and therefore, like the MI values, higher EPC values are of greater concern (Whittaker,2012). The strength, direction and significance of relationships between constructs and between covariance terms were assessed using the regression coefficients $(\beta)$ and $p$-values.

Mediation analysis (laccobucci et al., 2007) was used to evaluate indirect effects of perceived knowledge on behaviour intention, mediated by the other constructs.

Intercepts obtained from the SEM output provide a general idea of the sample population's response to the questions included in the final model, where 7 is strongly agree on the Likert scale or the most positive response on the bipolar scales and 1 is strongly disagree on the Likert scale or the most negative response on the bipolar scales. An intercept of 1-3 would therefore indicate a negative response, 4 a neutral response and 5-7 a positive response.

\section{Target sample size}

A subject to item ratio of 10:1 (i.e. 10 respondents for each question in the survey) can be used as a rule of thumb for determining the sample size needed for EFA, although $20: 1$ is preferable to minimise misclassification of items on the wrong factor (Costello and Osbourne, 2005). Therefore, a minimum sample size of 400 valid responses was required for EFA in this survey. There appears to be no consensus on sample size and power calculations for SEMs. Weston and Gore (2006) suggest a minimum sample size of 200 .
Commented [FV1]: Theory trumps statistics, so you should always consider this first. But if it's not intervening, you can modify the model using the MI, although not too much. 


\section{Results}

\section{Description of the population}

A total of 1451 responses were recorded between the $18^{\text {th }}$ December 2015 and $31^{\text {st }}$ January 2016; 873 were retained for further analysis. 422 (29.08\%) responses were incomplete and were removed from further analysis. Respondents were allowed to complete the survey multiple times to account for individuals that own or are employed at several premises. Therefore, responses with the same IP address (i.e. completed on the same computer or mobile device) were checked to ensure the responses pertained to different premises or owners and were not simply duplicate entries. One duplicate entry was removed from analysis as the respondent had completed all mandatory questions on both occasions but had only completed additional optional questions in one response. The full response was retained in this instance. The time spent completing the survey was checked for each remaining respondent to eliminate potential automated responses. The survey should have taken approximately 5-10 minutes to complete. A total of 149 responses were removed due to implausibly short completion times of less than one minute and consistent selection of the midpoint of the Likert and bipolar scales throughout the survey. Finally, postcodes were checked for validity using the UK grid reference finder (http://www.gridreferencefinder.com/postcodeBatchConverter/), which was unable to find 15 postcodes. Ten of these were found to be valid postcodes for Northern Ireland and the Channel Islands after further searches. The remaining 5 responses had either invalid or missing postcodes and were excluded from further analysis. 
255 Most respondents (93.58\%) were private horse or pony owners (Table 1).

256 Respondents reported a median of 2 horses and/or ponies (range 1-70) under their 257 care and were distributed throughout the UK and Channel Islands (Figure 2). 


\section{External validation}

The activity structure of the commercial respondents and the UK equine industry was not significantly different from that reported in the Lantra equine business survey (Lantra, 2011; $x^{2}=42$, d.f. $=36, p=0.227$ ). The regional distribution of commercial respondents and the distribution of UK equine businesses reported by Lantra (2011) was not significantly different $\left(x^{2}=12\right.$, d.f. $\left.=9, p=0.213\right)$. The distribution of respondents was broadly comparable with the distribution of horse owners in Great Britain in 2011 (Boden et al., 2012). However, quantitative comparison with this dataset was not possible.

\section{Internal validation}

All constructs were found to have good internal consistency with $\alpha>0.7$ but three factors could be improved by deleting items $x_{9}, x_{16}$ and $x_{33}$ (Appendix B, Table B1). These items were removed from subsequent analysis.

Validity of the perceived knowledge and behaviour intention constructs was confirmed using EFA specifying a single factor (Appendix B, Table B2 and B4). Items $\mathrm{X}_{31}$ and $\mathrm{x}_{32}$ underlying the subjective norms (norms) construct loaded weakly (factor loading $<0.3$ ) onto several factors (Appendix B, Table B3). Furthermore, items $\mathbf{x}_{10}-\mathrm{X}_{15}$ underlying the perceived severity of anthelmintic resistance construct loaded onto two factors, and items $\mathrm{x}_{19}-\mathrm{X}_{24}$ underlying the perceived severity of infection construct showed some weaker cross-loading onto several factors, suggesting a split between responses to the questions on the perceived severity of AR and infection in the context of horse health $\left(\mathrm{x}_{10}-\mathrm{x}_{12}\right.$ and $\left.\mathrm{x}_{22}-\mathrm{X}_{24}\right)$ and performance $\left(\mathrm{x}_{13}-\mathrm{x}_{15}\right.$ and $\left.\mathrm{x}_{19}-\mathrm{x}_{21}\right)$. Therefore, subsequent analyses compare the full model based on the proposed 
structural model (Figure 1), and a split model whereby the perceived severity of AR and perceived severity of infection constructs were divided to account for potential differences in responses to questions regarding animal health and performance.

\section{Structural equation modelling (SEM)}

SEM regression equations for the full model followed the structural model in Figure

1. Additional covariance terms were introduced between the constructs measuring perceived risk, and between construct measuring perceived control and subjective norms (Appendix C). SEM regression equations for the split model were the same as for the full model except that the perceived severity of AR and perceived severity of infection constructs were divided into two constructs each for reasons described above (Appendix C). Constructs were defined using the measurement model described in Appendix A.

The full model was re-specified to remove covariance between the following constructs as the covariances were not statistically significant (AppendixC): subjective norms and behavioural control, perceived susceptibility to infection and attitudes towards FECs, perceived severity of infection and attitudes towards anthelmintics, and perceived severity of AR and attitudes towards anthelmintics. Several Mls greater than the threshold value of 3.84 were identified, but in all cases the EPC was low, and some of the modifications were already captured in the model as covariances. Therefore the model was considered correctly specified. Specifically, covariance between subjective norms and perceived susceptibility to infection yielded a high MI of 15.767 but a low EPC of 0.221 . Similarly, covariance between subjective norms and attitudes towards FEC yielded a high $\mathrm{MI}$ and low EPC $(\mathrm{MI}=$ 
16.029, EPC $=0.17)$. Mls suggested that perceived susceptibility to infection may be dependent on the intention to use FECs $(\mathrm{MI}=4.072$, EPC $=0.123)$, subjective norms $(\mathrm{MI}=15.767, \mathrm{EPC}=0.165)$ and perceived behavioural control $(\mathrm{MI}=7.593$, EPC $=-0.278)$. However, the corresponding EPCs were low and therefore these changes were not made. Mls also suggested that attitudes towards anthelmintics may be dependent on perceived susceptibility to anthelmintic resistance $(\mathrm{MI}=7.813$, $\mathrm{EPC}=0.771)$, perceived severity of anthelmintic resistance $(\mathrm{MI}=6.775, \mathrm{EPC}=$ $0.270)$, perceived susceptibility to infection $(\mathrm{MI}=7.672, \mathrm{EPC}=1.318)$, perceived severity of infection $(\mathrm{MI}=7.053, \mathrm{EPC}=0.209)$, and attitudes towards FECs $(\mathrm{MI}=$ 7.388, EPC $=1.470)$. These modifications were not made as the model either already included terms for covariance between these variables or the covariances were removed as described above due to non-significance, and the EPC was low in all cases. A high MI suggested that attitudes towards FECs may be dependent on subjective norms $(\mathrm{MI}=16.029, \mathrm{EPC}=0.126)$ but the low EPC did not justify modification of the model. Finally, high Mls suggested that subjective norms may be dependent on perceived susceptibility to anthelmintic resistance $(\mathrm{MI}=11.345$, EPC $=0.116)$, severity of anthelmintic resistance $(\mathrm{MI}=7.583, \mathrm{EPC}=0.190)$, susceptibility to infection $(\mathrm{MI}=23.041, \mathrm{EPC}=0.177)$, severity of infection $(\mathrm{MI}=12.457, \mathrm{EPC}=$ $0.185)$ and attitudes towards FECs $(\mathrm{MI}=20.621, \mathrm{EPC}=0.242)$, but again, EPC values were low and therefore the modification was not justified. The full Ml output is provided in Appendix D.

The full SEM explained $50.6 \%$ of the variability in the intention to use FEC-TT/FECTST $\left(R^{2}=0.506 ; X^{2}=6186, d f=601, p<0.001\right)$. Intercepts from the full SEM output indicate that respondents had a moderately positive response to the questions relating to perceived knowledge (range $4.5-6.2$ ). On average, respondents had only 
a slightly positive response to the questions regarding perceived susceptibility to AR (range 4.54 - 4.57; i.e. they only slightly agreed that they were susceptible to the

334 development of AR), but gave a moderately positive response to questions regarding 335 the perceived severity of AR (range $5.51-5.88$; i.e. they agreed that AR was a 336 threat to horse health and performance).

337 Respondents gave a slightly negative response to the questions regarding susceptibility to infection (range 3.1-3.18; i.e. they slightly disagreed that their horses were susceptible to disease caused by worms), but gave a moderately positive response to questions regarding the severity of infection (range $5.48-5.77$; i.e. they agree that worms are a threat to horse health and performance).

Respondents reported slightly positive attitudes towards the use of anthelmintics (range $4.87-4.89$ ) and strong positive attitudes towards the use of FECs prior to treatment (range $6.42-6.51$ ). On average, there was only a slightly positive

345 response to the questions regarding subjective norms (range $4.04-4.75$ ). They also 346 gave moderately positive responses to the questions regarding behavioural control 347 (range $5.96-6.2$; i.e. they agreed that the decision to use FECs and treat their horses was under their control and that they could obtain FECs). Finally, on average, respondents reported a moderately positive response to the intention to use FECs in future (range 5.29 - 5.63). The intercepts are detailed in the SEM output in Appendix D. 
All specified covariances were statistically significant and all items were significantly associated with the assigned constructs. Full output and standard fit indices are reported in Appendix D. There was a significant, but weak, positive influence of the perceived susceptibility of AR on the intention to use FECs before treating. None of the other perceived risk constructs were significantly associated with intention to use FECs. A more positive attitude towards anthelmintics was weakly associated with a decrease in intention to use FECs. A more positive attitude towards FECs, an increase in societal/peer influences (subjective norms) and an increase in perceived control were strongly associated with an increased intention to use FECs (Table 2).

There was no direct effect of perceived knowledge on the intention to use FECs before treating (Tables 2 and 3). However, there was a significant indirect influence of perceived knowledge on behaviour intention via several mediating constructs (Table 3). An increase in perceived knowledge was associated with a more negative attitude towards anthelmintics (Table 2) which in turn led to an increase in the intention to use FECs (Tables 2 and 3). However, the overall effect of perceived knowledge on behaviour intention mediated by attitudes towards anthelmintics was much weaker than for other mediating constructs. There were stronger, significant positive influences of perceived knowledge on behaviour intention mediated by attitudes towards FECs, subjective norms and perceived control (Tables 2 and 3). The total indirect effect of knowledge on the intention to use FECs $(\beta=0.76$, Odds Ratio $(\exp (\beta))=2.14$; Table 3 ) was greater than the direct effect of any single construct (Table 3).

The split SEM yielded an identical outcome to the full SEM, albeit with slightly different coefficients (Appendix D). The standard fit indices and AIC were slightly 
improved $(\mathrm{AIC}($ full $)=83737, \mathrm{AIC}($ split $)=80499)$ but the split model was rejected in favour of the more parsimonious full model.

\section{Discussion}

The adoption of new animal health management strategies and technology requires animal owners to make complex risk assessments. Animal owners must also work within the practical limitations of their particular management system and available resources. Furthermore, as non-experts, animal owners must assess risk based on incomplete knowledge of the health issue, management strategies and scientific evidence base. The framework described here, based on the Theory of Planned Behaviour and the Health Belief Model, captures these issues by measuring perceived risk, attitudes towards FECs and the use of anthelmintics, subjective norms and perceived control.

Socio-cognitive models such as the TPB and HBM are statistically testable frameworks which can be used to determine which factors influence behavior. However, all have their limitations, for example the exclusion of unconscious influences and emotion, habits and the translation of intention into behaviour (Sniehotta et al., 2014) and there is scope for factors not included in the model such as emotion to influence the behaviour intention. These socio-cognitive theories can therefore be framed as a part of a bigger picture, the static and more rational part of behaviour (Vande Velde et al., 2017).

The intention of respondents to use FEC-TT or FEC-TST in future (i.e. conduct a FEC prior to treating groups or individual horses, respectively) was evaluated as 
'behaviour intention' in the framework. Attitudes towards diagnostics (FECs) and anthelmintics were included as constructs to evaluate on the one hand the 'attitude towards the behaviour' component of the TPB, and on the other hand the 'benefits barriers' component of HBM as described by Vande Velde et al., (2015). 'Subjective norms' and 'perceived behavioural control' constructs were also evaluated as described by Vande Velde et al., (2015).

'Perceived risk' was evaluated using constructs measuring 'perceived susceptibility to AR' and 'perceived severity of AR' as described by Vande Velde et al., (2015). However, many equids in the UK are considered companion animals and, as GIN infection may lead to acute disease such as colic (Corning, 2009), it is possible that fear of disease may drive horse owners to treat prophylactically regardless of cost or future development of $A R$ and to avoid selective treatment strategies. Therefore the framework was extended to include additional constructs evaluating perceived risk of infection (susceptibility and severity) in addition to the perceived risk of AR (Figure 1). Additional items evaluating 'perceived severity of infection' and 'perceived severity of AR' in the context of horse performance were also included to reflect the potentially divergent priorities of different sectors of the equine industry. These four constructs were allowed to covary as they are components of the shared perceived risk measure.

Similar studies on livestock have shown that the farmer's assessment of the efficacy of the strategy in question is an important consideration (Valeeva et al., 2011;

Garforth et al., 2013), that access to sufficient, trusted information may influence farmer behaviour with regards to biosecurity and animal health (Toma et al., 2013), and that knowledge and awareness of practices may influence behaviour intention (Garforth et al., 2013). Furthermore, an individual's knowledge and awareness may 
affect the perceived credibility of peer and societal influences, and affect their perceived control (how can one be in control of something that they do not understand?). It therefore follows that a horse owner's intention to use FEC-TT or FEC-TST may be driven in part by their knowledge and access to information. The framework was therefore extended to include the horse owners' perceived knowledge as a construct which may directly or indirectly affect behaviour intention (Figure 1).

The significant $\mathrm{X}^{2}$ statistic obtained here was at odds with good model performance in the structural evaluation and the model was therefore accepted. The SEM output and subsequent conclusions were very robust to changes in the structural model (indicated by the modification indices and comparison of the full and split models) and were consistent with similar surveys conducted in other livestock sectors in Europe (Vande Velde et al., 2015). This dual approach to model evaluation was undertaken as the $\mathrm{X}^{2}$ test has been criticized for its sensitivity to large sample sizes and susceptibility to type I errors (Weston and Gore, 2006; Barrett, 2007; Saris et al. 2009). Furthermore, it does not provide any indication of the suitability of the structural model and misclassification errors. Therefore, evaluation of modification indices has been suggested as an alternative method for model evaluation (Saris et al., 2009).

The perceived risk of anthelmintic resistance and GIN infection, measured using constructs related to perceived susceptibility and severity of resistance and infection in the context of both horse health and performance, was surprisingly not associated with the intention to use FECs. Therefore, the initial assumption that perceived risk levels may drive the adoption of FECs is not supported. Vande Velde et al., (2015) found a similar pattern in Belgian dairy cattle farmers' attitudes towards diagnosis 
before GIN treatment, but this was attributed to the relatively low levels of anthelmintic resistance in dairy cattle in Western Europe. In contrast, anthelmintic resistance is a common and well-publicised problem in UK horse populations, where sub-optimal efficacy of all three classes of anthelmintics (macrocyclic lactones, pyrantel and benzimidazoles) against cyathostomins has been detected (Relf et al., 2014) and sub-optimal efficacy of fenbendazole has been detected on $100 \%$ of yards tested (Lester et al., 2013; Relf et al., 2014; Stratford et al., 2014).

It is interesting to note that, overall, respondents thought that their horses were not susceptible to disease caused by GINs but agreed that GIN infection was a threat to horse health and performance. The low perceived risk of GIN infection may be due to the chronic nature of the majority of clinical infections and lack of awareness of acute larval cyathostominosis.

Similarly, the perceived severity of anthelmintic resistance was greater than the perceived susceptibility to anthelmintic resistance, suggesting that respondents thought that AR was a significant general issue but that they were less susceptible than others. This attitude is also prevalent in sheep farmers (Morgan and Coles, 2010; Morgan et al., 2012) and could possibly be addressed inexpensively by regular monitoring of pre- and post-treatment FECs to demonstrate the local status and risks of infection and AR. However, the results here suggest that failure to adequately assess risk of infection and AR are not barriers to the uptake of FEC-TT/FEC-TST and therefore efforts may be better directed elsewhere.

There was a mildly positive overall attitude towards the use of anthelmintics to prevent GIN infection. This suggests that horse owners consider prophylactic treatment to be effective and perhaps explains the relatively low perceived 
susceptibility to anthelmintic resistance regardless of the high prevalence of $A R$ in UK horse populations (Lester et al., 2013; Relf et al., 2014; Stratford et al., 2014). Furthermore, a negative attitude towards the prophylactic use of anthelmintics was weakly associated with greater intention to use FECs prior to treatment, suggesting that owners wishing to move away from anthelmintic use for any reason (e.g. in favour of using 'herbal' wormers) are more likely to use FECs.

Consistent with Vande Velde et al., (2015), a positive attitude towards diagnostics (FECs) was strongly associated with a greater intention to use FECs prior to treatment. Vande Velde et al., (2015) found that attitudes towards diagnostics were more positive for dairy cows than calves, perhaps due to the ease of implementation and lower costs of bulk milk tank ELISAs used in dairy cows compared with the FECs used in calves. The current survey did not explore the motivations and opinions underlying respondents' attitudes towards FECs and further work is necessary to elucidate these factors.

Subjective norms were a strong driver of intention to use FEC-TT/FEC-TST, indicating the importance of peers and advisers in the decision-making process. In this respect, the recent release of equine anthelmintic prescribing protocols that promote the use of FECs in the UK is a step in the right direction. Privately owned horses and ponies are typically kept in small groups on private land, or on liveries where several owners keep their horses on the same yard and where the deworming strategy of one owner directly affects the health of other horses on the yard.

Therefore, advocates for FEC-TT/FEC-TST on livery yards or shared grazing may present an opportunity to encourage more widespread use of FECs through peer influence. 
Greater perceived control of deworming strategy was also strongly associated with a greater intention to use FECs prior to treatment. This, in contrast to the lack of effect of perceived risk, demonstrates the importance of self-efficacy and control in health related behaviours - regardless of the perceived risk, an individual can only perform a behaviour if they believe they have control over their circumstances and ability to perform the behaviour. Access to suitable FEC services will undoubtedly play a key role, but other fundamental barriers exist which limit perceived control. For example, in some cases respondents commented that there was a lack of control over deworming at the livery yard where their horse was kept. In other cases it may be that a lack of understanding translates to a lack of perceived control. In a study of pesticide safety behaviour in Mexican farmworkers in the US, which was also based on the HBM framework, access to information on pesticide safety increased subjects' perceived control of behaviours associated with limiting exposure to pesticides (Arcury et al., 2002). Arcury et al., further suggest that education and demonstrations aimed at increasing self-efficacy would lead to behaviour change. On the other hand, livery yard managers that dictate yard worm control strategies could have a positive role in co-ordinating deworming activities in co-grazing horses for the greater good, to include considerations of sustainability.

The importance of education and awareness in empowering behaviour change is reflected in the mediation analysis. Attitudes towards FECs and anthelmintics, subjective norms and perceived control were all directly influenced by an increase in perceived knowledge, which had a strong positive indirect effect on behavior intention. Therefore, even small increases in the perceived knowledge of horse owners could be beneficial to encourage sustainable nematode control practices. Respondents were confident in the statement that they knew what worm egg counts 
were for and that they knew enough about worms and deworming to decide on an appropriate worm control strategy, but were less confident in their knowledge of the limitations of worm egg counts and their ability to interpret worm egg counts without the help of an adviser. Garforth et al., (2013) note that 'the fact that someone knows about a measure and understands what it is designed to do does not make it more likely that they will implement it' and that the comments of farmers in their survey suggested that practicability and the ability to assess the efficacy of the measure are important considerations.

In the present study, some horse owners commented on the quality, amount, and impartiality of information available to them. Some horse owners also demonstrated clear misunderstandings of gastrointestinal nematode biology, the epidemiology of disease and the limitations of FECs, which further demonstrate the importance of communicating the limitations of egg counts and other sustainable control strategies to users. They may also reflect a wider problem of companies offering potentially inaccurate FEC services based on inadequate faecal samples, damaging the credibility of FEC-based deworming strategies as some respondents communicated concerns and distrust with regards to the sampling methods used by commercial providers.

\section{Conclusions}

Based on the results of this survey and the comments of respondents, knowledge transfer activities focussing on increasing awareness and understanding, and delivering training on the practical implementation of FECs, may improve the uptake of sustainable parasite control practices such as FECs on equine holdings in the UK, 
547 primarily by improving self-efficacy and perceived control. It is, however, important

548 that such activities are supported by targeted research on the opinions and attitudes

549 of horse owners to current information sources and methods of knowledge transfer,

550 as opinions of livestock farmers on the credibility of information sources and the

551 translation of scientific evidence underpinning animal health measures was reported

552 to be variable (Garforth et al., 2013). Furthermore, emphasising the dangers of the

553 status quo, in this case nematode infection and the development of AR, may not

554 encourage behaviour change. Knowledge transfer activities should therefore focus

555 on improving self-efficacy through improved knowledge of the system and available

556 nematode control options, as well as identifying and addressing potential barriers to

557 the uptake of sustainable nematode control strategies. 


\section{Conflicts of interest}

HRV delivers training and knowledge exchange activities related to faecal egg counting and sustainable nematode control in livestock and horses. The research was conducted as part of a project aiming to develop automated parasite egg counting technology to support targeted treatment of grazing livestock. Funding details are provided in the acknowledgments.

\section{Acknowledgements}

This research was partly funded by InnovateUK and the UK Biotechnology and Biological Sciences Research Council (BBSRC) under project TS/L000784/1 AutoFEC. The authors would like to thank James Fletcher and Mike Fletcher for their comments on the survey content.

\section{References}

Ajzen, I., 2002. Constructing a TpB Questionnaire: Conceptual and Methodological Considerations, Retrieved from http://www.unibielefeld.de/ikg/zick/ajzen\%20construction\%20a\%20tpb\%20questionnaire.pdf (accessed 10.10.16)

Alarcon, P., Wieland, B., Mateus, A. L. P., Dewberry,C., 2014. Pig farmers' perceptions, attitudes, influences and management of information in the decisionmaking process for disease control. Prev. Vet. Med. 116, 223-242

Animal Health Distributors Association, 2015. SQP and Vet Anthelmintic Prescribing Protocol for Horses, Available on request from http://www.ahda.co.uk/ 
Archery, T. A., Quandt, S. A., Russell, G. B., 2002. Pesticide safety among farmworkers: perceived risk and perceived control as factors reflecting environmental justice. Env. Health Persp. 110, S2

Barrett, P., 2007. Structural equation modelling: adjudging model fit. Person. Individ. Diff. 42, 815-824

Boden, L. A., Parkin, T. D. H., Yates, J., Mellor, D., Kao, R. R., 2012. Summary of current knowledge of the size and spatial distribution of the horse population within Great Britain. BMC Vet. Res. 8, 43

Charlier, J., Morgan, E.R., Rinaldi, L., Van Dijk, J., Demeler, J., Hoglund, J., Hertzberg, H., Van Ranst, B., Hendrickx, G., Vercruysse, J., Kenyon, F., 2014. Practices to optimise gastrointestinal nematode control on sheep, goat and cattle farms in Europe using targeted (selective) treatments. Vet. Rec. 175, 250-255.

Corning, S., 2009. Equine cyathostomins: a review of biology, clinical significance and therapy. Parasites \& Vectors, 2: S1. doi:10.1186/1756-3305-2-S2-S1

Costello, A. B., Osbourne, J. W., 2005. Best practices in Exploratory Factor Analysis: four recommendations for getting the most from your analysis. Practical Assessment, Research \& Evaluation 10, Retrieved from http://pareonline.net/getvn.asp?v=10\&n=7 (accessed 10.10.16)

Easton S., Pinchbeck, T., Tzelos, T., Bartley, D. J., Hotchkiss, E., Hodgkinson, J. E., Matthews, J. B., 2016. Investigating interactions between UK horse owners and prescribers of anthelmintics Prev. Vet. Med. 135, 17-27

Francis, A.J.J., Eccles, M.P., Johnston, M., Walker, A., Grimshaw, J., Foy, R., Kaner, E.F.S., Smith, L., Bonetti, D., 2004. Constructing questionnaires based on the theory 
of planned behaviour, a manual for health service researchers. Centre for health

606 service research, University of Newcastle

607 Garforth, C. J., Bailey, A. P., Tranter, R. B., 2013. Farmers' attitudes to disease risk 608 management in England: A comparative analysis of sheep and pig farmers. Prev.

609 Vet. Med. 110, 456-466

610 Hooper, D., Coughlan, J., Mullen, M. 2008. Structural Equation Modelling: Guidelines

611 for Determining Model Fit. Electronic Journal of Business Research Methods, 6(1),

$61253-60$

613 laccobucci, D., Saldanha, N., Deng, X., 2007. A meditation on mediation: evidence

614 that structural equation models perform better than regressions. J. Cons. Psych. 17,

$615 \quad 140-154$

616 Jones, P. J., Marier, E. A., Tranter, R. B., Wu, G., Watson, E., Teale, C. J., 2015.

617 Factors affecting dairy farmers' attitudes towards antimicrobial medicine usage in

618 cattle in England and Wales. Prev. Vet. Med. 121, 30-40

619 Lantra, 2011. A study into the business and skills requirements of the UK equine

620 industry. Lantra, Warwickshire. Retrieved from: www.lantra.co.uk (accessed

$621 \quad \underline{10.10 .16)}$

622 Lester, H.E., Spanton, J., Stratford, C.H., Bartley, D.J., Morgan, E.R., Hodgkinson,

623 J.E., Coumbe, K., Mair, T., Swan, B., Lemon, G. and Cookson, R., 2013. Anthelmintic

624 efficacy against cyathostomins in horses in Southern England. Vet. Parasitol. 197,

$625 \quad 189-196$

626 Matthews, J., Lester, H., 2015. Control of equine nematodes: making the most of

627 faecal egg counts. In Practice, 37, 540-544 
Morgan, E.R., Coles, G.C., 2010. Nematode control practices on sheep farms following an information campaign aiming to delay anthelmintic resistance. Vet. Rec. $166,310-303$.

Morgan, E.R., Hosking, B.C., Burston, S., Carder, K.M., Hyslop, A.C., Pritchard, L.J., Whitmarsh, A.K., Coles, G.C., 2012. A survey of helminth control practices on sheep farms in Great Britain. Vet. J. 192, 390-397

Nielsen, M. K., Monrad, J., Olsen, S. N., 2006. Prescription-only anthelmintics-A questionnaire survey of strategies for surveillance and control of equine strongyles in Denmark. Vet. Parasitol. 135, 47-55

QGIS Development Team, 2015. QGIS Geographic Information System. Open Source Geospatial Foundation Project. http://qgis.osgeo.org (accessed 10.10.16

R Core Team, 2015. R: A language and environment for statistical computing. $R$ Foundation for Statistical Computing, Vienna, Austria. https://www.R-project.org/

Relf, V. E., Lester, H. E., Morgan, E. R., Hodgkinson, J. E. and Matthews, J. B., 2014. Anthelmintic efficacy on UK Thoroughbred stud farms. Int. J. Parasitol. 44, 507-514 Revelle, W. 2015. psych: Procedures for Personality and Psychological Research, |Northwestern University, Evanston, Illinois, USA, Retrieved from http://CRAN.Rproject.org/package=psych (accessed 10.10.16)

Rose, H., Rinaldi, L., Bosco, A., Mavrot, F., De Waal, T., Skuce, P., Charlier, J., Torgerson, P.R., Hertzberg, H., Hendrickx, G., Vercruysse, J., Morgan, E.R., 2015. Widespread anthelmintic resistance in European farmed ruminants: a systematic review. Vet. Rec. 176, 21, doi: 10.1136/vr.102982. 
Rosseel, Y. 2012. lavaan: An R Package for Structural Equation Modeling. Journal of Statistical Software, 48(2), 1-36. Retrieved from http://www.jstatsoft.org/v48/i02/.

Rosenstock, I.M., Strecher, V.J., Becker, M.H., 1988. Social learning theory and the health belief model. Health Educ. Behav. 15, 175-183

Sallé, G., Cabaret, J., 2015. A survey on parasite management by equine veterinarians highlights the need for a regulation change. Vet. Rec. Open, 2, e000104

Saris, W. E., Satorra, A., van der Veld, W. M., 2009. Testing structural equation models or detecting misspecifications?. Structural Equation Modeling: A Multidisciplinary Journal 16, 561-582

Sniehotta, F. F., Presseau, J., Araújo-Soares, V., 2014. Time to retire the theory of planned behaviour. Health Psychol. Rev. 8, 1-7.

Stratford, C. H., Lester, H. E., Pickles, K. J., McGorum, B. C. and Matthews, J. B, 2014. An investigation of anthelmintic efficacy against strongyles on equine yards in Scotland. Eq. Vet. J. 46, 17-24

Toma, L., Stott, A. W., Heffernan, C., Ringrose, S., Gunn, G. J., 2013. Determinants of biosecurity behaviour of British cattle and sheep farmers - a behavioural economics analysis. Prev. Vet. Med. 108, 321-333

Valeeva, N. I., van Asseldonk, M. A. P. M., Backus, G. B. C., 2011. Perceived risk and strategy efficacy as motivators of risk management strategy adoption to prevent animal diseases in pig farming. Prev. Vet. Med., 102, 284-295

Vande Velde, F., Claerebout, E., Cauberghe, V., Hudders, L., Van Loo, H., Vercruysse, J., Charlier, J., 2015. Diagnosis before treatment: Identifying dairy 
673 farmers' determinants for the adoption of sustainable practices in gastrointestinal

674 nematode control. Vet. Parasitol. 212, 308-317.

675 Vande Velde, F., Charlier, J., Cauberghe, V., Hudders, L., Claerebout E., 2017. Re-

676 Reconceptualising the 'Behavioural Approach' in Agricultural Studies: Beyond a

677 Cognitive Socio-Psychological Perspective. International Society for Economics and

678 Social Sciences of Animal Health, 27-28 March 2017, Aviemore, UK

679 Weston, R., \& Gore, P. A., Jr. 2006. A brief guide to structural equation modeling.

680 The Counseling Psychologist, 34, 719-751.

681 Whittaker, T. A., 2012 Using the Modification Index and Standardized

682 Expected Parameter Change for Model Modification, J. Experimental Education, 80,

$683 \quad 26-44$. 